\title{
Effectiveness of negative pressure wound therapy for the wound of ileostomy closure: a multicenter, phase II randomized controlled trial
}

Koichiro Kojima ${ }^{1 *}$, Mayu Goto ${ }^{2}$, Yasuo Nagashima ${ }^{2}$, Yoko Saito ${ }^{3}$, Masaya Kawai $^{4}$, Shihori Takebe ${ }^{5}$, Akiko Egawa ${ }^{6}$, Mitsuko Tanba ${ }^{7}, K^{\prime}$ Kazue Ishikawa ${ }^{8}$, Hiroyoshi Matsuoka ${ }^{9}$, Tadahiko Masaki ${ }^{1}$, Eiji Sunami ${ }^{1}$, Norihiko Ohura $^{10}$, Koji Teruya ${ }^{11}$, Ken Eto ${ }^{12}$, Keijiro Nozawa ${ }^{13}$, Kazuhiro Sakamoto ${ }^{4}$ and Kimihiko Funahashi ${ }^{2}$

\begin{abstract}
Background: The American Society of Surgery and American Society for Surgical Infections issued guidelines for surgical site infections (SSIs) in December 2016. These guidelines recommend a purse-string suture (PSS) for stoma closure as it facilitates granulation and enables open wound drainage. This study investigated the effect of using negative pressure wound therapy (NPWT) along with standard PSS and aimed to determine the optimal period of NPWT use.
\end{abstract}

Methods: The patients were divided into three groups as follows: Group A, postoperative wound management alone with gauze exchange as the representative of conventional PSS; Group B, the performed management was similar to that of Group A plus NPWT for 1 week; and Group C, the performed management was similar to that of Group A plus NPWT for 2 weeks. Regarding objective measures, the wound reduction rate was the primary outcome, and the incidence of SSIs, length of hospital stay, and wound healing duration were the secondary outcomes.

Results: In total, 30 patients (male: 18, female: 12) were enrolled. The average age was 63 (range: 43-84) years. The wound reduction rate was significantly higher in Group B than in Group A on postoperative days (PODs) 7 (66.1 vs. 48.4\%, $p=0.049$ ) and 10 (78.6 vs. 58.2\%, $p=0.011$ ), whereas no significant difference was observed on POD 14. Compared with Group A, Group C (POD 7: 65.9\%, POD 10: 69.2\%) showed an increase in the wound reduction rate on POD 7, although the difference was not significant $(p=0.075)$. SSIs were observed in Groups B $(n=2)$ and C $(n=2)(20 \%)$ but not in Group A (0\%).

Conclusions: The most effective duration of NPWT use for ileostomy closure with PSS in terms of the maximum wound reduction rate was from PODs 3 to 10. However, NPWT did not shorten the wound healing duration. NPWT may reduce the wound size but should be used with precautions for SSIs. The small sample size (30 cases), the use of only one type of NPWT system, and the fact that wound assessment was subjective and not blinded were the limitations of this study. Further studies are needed to confirm our findings.

\footnotetext{
*Correspondence: k-kojikoji@ks.kyorin-u.ac.jp

${ }^{1}$ Department of Surgery, Kyorin University School of Medicine, 6-20-2,

Shinkawa, Mitaka city, Tokyo 181-8611, Japan

Full list of author information is available at the end of the article
}

(C) The Author(s) 2021. Open Access This article is licensed under a Creative Commons Attribution 4.0 International License, which permits use, sharing, adaptation, distribution and reproduction in any medium or format, as long as you give appropriate credit to the original author(s) and the source, provide a link to the Creative Commons licence, and indicate if changes were made. The images or other third party material in this article are included in the article's Creative Commons licence, unless indicated otherwise in a credit line to the material. If material is not included in the article's Creative Commons licence and your intended use is not permitted by statutory regulation or exceeds the permitted use, you will need to obtain permission directly from the copyright holder. To view a copy of this licence, visit http://creativecommons.org/licenses/by/4.0/. The Creative Commons Public Domain Dedication waiver (http://creativeco mmons.org/publicdomain/zero/1.0/) applies to the data made available in this article, unless otherwise stated in a credit line to the data. 
Trial registration: UMIN Clinical Trials Registry; UMIN000032174 (10/04/2018).

Keywords: Negative pressure wound therapy, lleostomy closure, Wound healing, Surgical site infection, Purse-string suture, Randomized controlled trial, Phase II trial

\section{Background}

In recent years, the widespread adoption of laparoscopic and robot-assisted surgeries and development of advanced surgical techniques and devices have enabled lower anastomosis during rectal cancer surgery. Although anal preservation has become possible, a temporary stoma is often constructed to prevent anastomotic sepsis in many cases, such as when the anastomosis is close to the anus. A temporary stoma that is constructed to prevent the complications of colorectal surgery is closed 3-6 months after the initial surgery. The complication rate can be as high as $20-40 \%$, with the most common complication being surgical site infections (SSIs), as the wound of stoma closure is at a site where the feces was expelled [1-3].

Since 1995, purse-string suture (PSS) for stoma closure has been reported to be useful for preventing SSIs, mainly in Europe and the United States. PSS for stoma closure is a method of suturing the dermis in an annular shape to create a semi-open wound, that significantly reduces the incidence of SSIs compared with the conventional simple wound closure $[4,5]$. The incidence rate of SSI with this method was reported to be 0-5\% [6]. Recently, guidelines in Europe and the United States recommended PSS as a method of wound closure following stoma creation [7]. In Japan, PSS is the standard treatment in several institutions; however, it requires approximately 1 month for complete wound healing. Therefore, the wound of stoma closure with PSS is considered to be an intractable wound. There are some problems associated with wound management post-PSS, including the need for regular wound cleaning and replacement of gauze, as well as the cost of treatment tools. Furthermore, patients are often discharged from the hospital before achieving complete wound healing; hence, monitoring the wound and expenditure related to the medical materials needed for its management become the patients' responsibility.

By shrinking the wound edges, increasing blood flow, promoting granulation, reducing edema, and removing excess exudates, negative pressure wound therapy (NPWT) promotes wound healing through the application of negative pressure to wounds, such as pressure ulcers, acute traumatic wounds, chronic intractable wounds, and postoperative wound infections [8-11]. It has been widely used in Europe and the United States since 1995 and has been covered by the medical insurance of the Ministry of Health, Labor, and Welfare of
Japan since 2010. Recently, the efficacy of incisional NPWT (iNPWT) for the prevention of wound complications in closed surgical incisions has been reported [12]. However, in this study, the wound was not completely closed but partially opened. Additionally, NPWT was used to promote granulations of the subcutaneous tissue, which is considered different from iNPWT. Therefore, the combined use of NPWT with stoma closure is expected to facilitate wound healing by increasing wound shrinkage, reducing the wound healing time and SSI incidence, and reducing the personal and economic burden on medical staff, patients, and caretakers.

Several case reports and retrospective studies evaluating the effectiveness of NPWT for stoma closure have reported a shorter wound healing duration and lower incidence of SSIs using NPWT [13-16]. Azuma et al. reported that NPWT for stoma closure significantly reduced the average wound volume from $2.3 \mathrm{~mL}$ on the day of surgery to $0.16 \mathrm{~mL}$ on postoperative day (POD) 7 in 20 cases. Naito et al. reported that the incidence rate of wound infection was $6.7 \%$ and the average wound healing duration was 18 days in 30 cases. Moreover, compared with previous reports on PSS without NPWT, the incidence rate of wound infection was expected to decrease, while the wound healing duration was expected to shorten. Obeid et al. reported that NPWT for stoma closure reduced the frequency of visits to the dressing clinic, suggesting a significant implication for cost savings [15]. There is only one reported of a prospective single-center randomized controlled trial [17]; however, it did not report a significant effect on the complete wound healing duration and incidence of SSIs. Additionally, there are no reports of multicenter studies. The effectiveness of combined NPWT with stoma closure has not yet been established. In addition, factors, such as the size of foam, time of insertion, and the duration of dressing, are unclear. Therefore, in this prospective, multicenter randomized controlled trial, we aimed to explore the effectiveness and optimal duration of NPWT use for stoma closure.

\section{Methods \\ Patients}

This prospective randomized controlled trial was conducted between March 2018 and March 2019 at five university hospitals in Tokyo, Japan. We enrolled patients with an ileostomy who were scheduled for closure surgery and obtained their written consent for 
participation in the study after explaining them the purpose of the study using a form approved by the ethics committee of each institution. The exclusion criteria were as follows: the presence of comorbidities that were strongly suspected to affect wound healing, such as familial adenomatous polyposis or inflammatory bowel disease; hemodialysis; poorly controlled diabetes mellitus (glycated hemoglobin level of $\geq 7.5 \%$ ); steroid medications; and difficulty in changing dressings due to dementia, psychiatric illness, or postoperative delirium. The patients were randomly assigned to the treatment groups post-enrollment by the central allocation method. For the random assignment, every case was simply randomized at an allocation ratio of $1: 1: 1$, and the random sequences were generated using a random number table associated with the statistical accountability of this study. The trial was not interrupted, and enrollment was terminated when the expected number of patients was reached.

\section{Surgical techniques (PSS)}

After closure of the peritoneum and rectus abdominis sheath during the stoma closure surgery, the dermis and subcutaneous tissue were sutured in a ring with 6-12 sutures using a monofilament absorbent suture (1 PDS Plus, CTB $40 \mathrm{~mm} \mathrm{1/2} \mathrm{Circle;} \mathrm{Ethicon} \mathrm{Inc.,}$ Somerville, NJ, USA). Then, it was ligated with a $5-\mathrm{mL}$ syringe, creating an open wound with a diameter of approximately $15 \mathrm{~mm}$ for drainage. After surgery, the wound was protected with a dressing sheath that was removed the following day (Fig. 1).

\section{Wound management} Group A (control group)

The dressing was removed on POD 1, and the wound was washed daily thereafter. Moreover, the gauze was mainly used and changed repeatedly until epithelialization occurred.

\section{Group B (NPWT weekly-use group)}

The day after surgery, the dressing was removed, and a foam insert measuring $6 \times 6 \times 20 \mathrm{~mm}$ was introduced into the wound after wound cleaning. The foam was cut from Smith \& Nephew Foam Filler (Smith \& Nephew Co. Ltd., London, United Kingdom). Subsequently, NPWT (PICO Single Use Negative Pressure Wound Therapy System; Smith \& Nephew Co. Ltd.), which operates at a negative pressure of $80 \mathrm{mmHg}$, was initiated. On POD 3, the dressing and foam were removed. After wound cleaning, NPWT was continued without inserting a foam piece into the wound. NPWT was completed on POD 7, and appropriate wound cleaning was performed. The gauze was changed until epithelialization occurred, similar to the procedure performed in the control group (Fig. 2).

\section{Group C (NPWT fortnightly use group)}

The day after surgery, the dressing was removed, and a similar foam to that used in Group B was introduced into the wound after wound cleaning. Subsequently, NPWT, which operates at a negative pressure of $80 \mathrm{mmHg}$, was initiated. On POD 3, the dressing and foam were removed. After wound cleaning, NPWT was continued without inserting a foam piece into the wound. On PODs 7 and 10, the dressings were removed, wound cleaning was performed, and NPWT was continued. NPWT was completed on POD 14, and appropriate wound cleaning

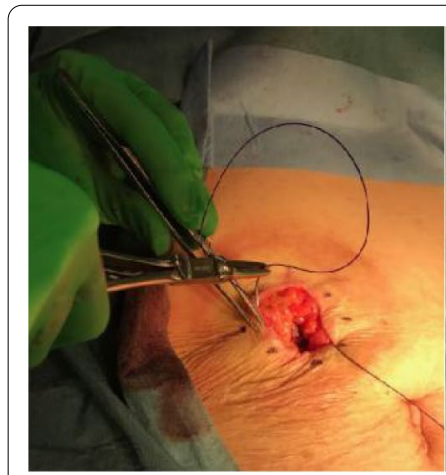

Suture the dermis and subcutaneous area in an annular fashion with 6-12 markings on the skin

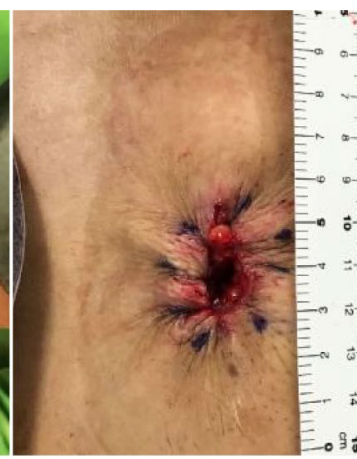

Create an open wound of approximately $15 \mathrm{~mm}$

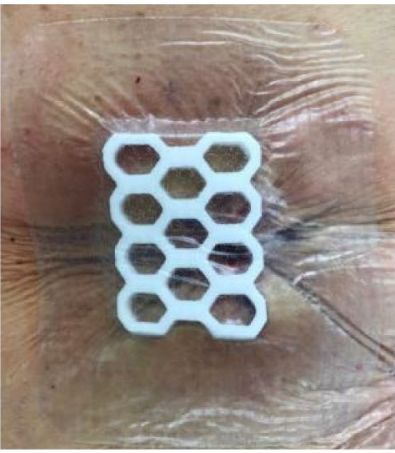

Protect the wound with a dressing after the surgery

Fig. 1 Purse-string suture. The performed method of PSS is presented. We used 1 PDS Plus as the suture and a 5-mL syringe was used to create an open wound. PSS purse-string suture 


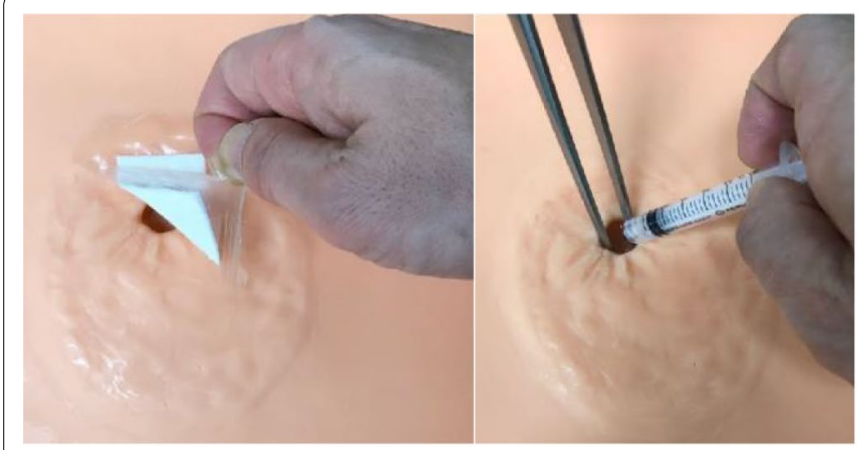

Remove dressing
Wash the wound

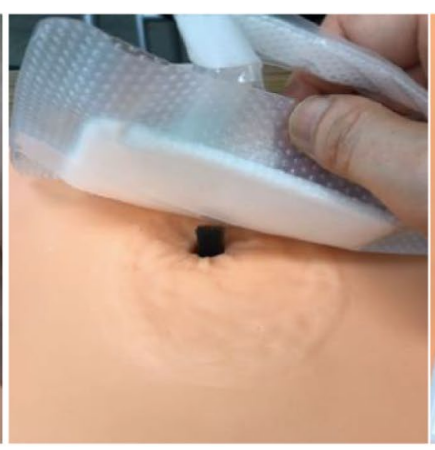

Insert the foam into the wound and attach the dressing

* The foam size: $6 \times 6 \times 20 \mathrm{~mm}$

Remove the foam at POD 3

Fig. 2 Wound treatment in Groups B and C. The wound management method using the PICO system for PSS is presented. The foam inserted when applying PICO was removed on POD 3. PSS purse-string suture, POD postoperative day

was performed. The gauze was changed until epithelialization occurred, similar to the procedure in the control group; however, if exudates were not observed to adhere to the dressing while changing it on PODs 7 and 10, these were considered the dates of wound healing (Fig. 2).

\section{Endpoint criteria}

The primary endpoint was selected as the rate of wound reduction from the original wound volume. The incidence of SSI, wound size (long diameter $\times$ short diameter, depth), healing time, and complication rate (excluding SSI) were also evaluated. The wound volume was measured on PODs 1, 3, and 7 and compared with that on POD 1 to calculate the reduction rate. To measure the wound volume, a saline solution was injected into the wound, and the volume was calculated based on the amount of fluid retained (Fig. 3). Wound healing was defined as the absence of exudates adhering to the gauze or skin protector applied to the wound. SSI was diagnosed if pus was observed in the wound. SSIs were classified as superficial incisional, deep incisional, and organ/ space SSIs, depending on the site of infection.

\section{Statistical analyses}

Continuous data are presented as medians and ranges. Comparative analysis of continuous variables was performed using the Mann-Whitney $U$ test. The test for three independent sample data is non-parametric; therefore, the Kruskal-Wallis test was used for this analysis. Categorical variables were compared using the chi-square and Fisher's exact tests. The level of significance was set at $\mathrm{p}<0.05$.

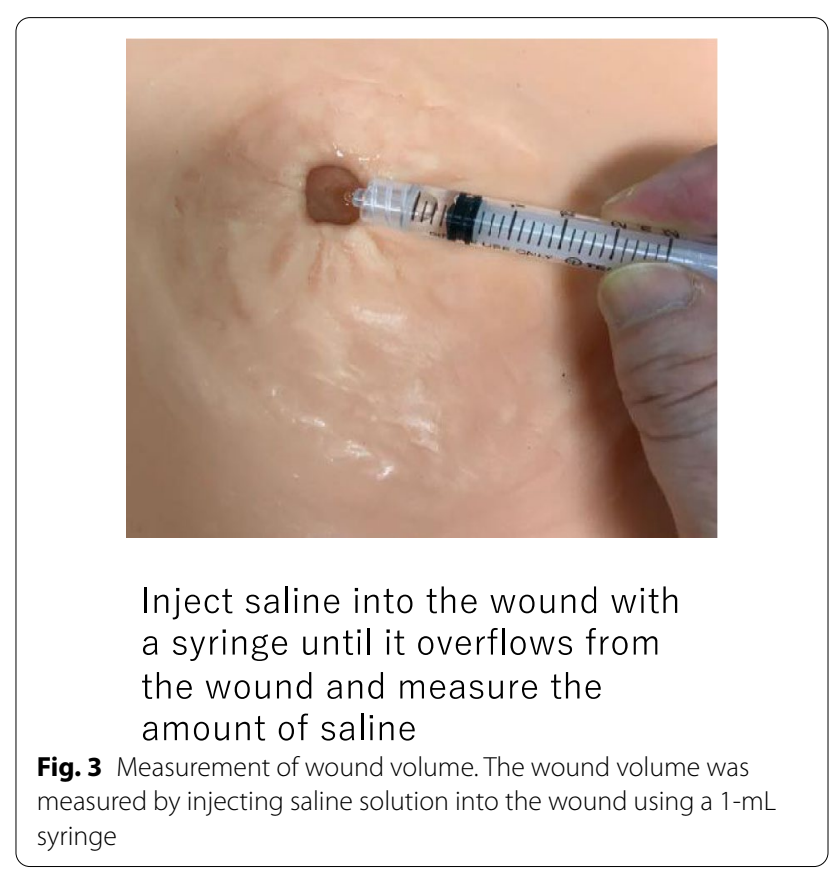

Before conducting the study, an acceptable sample size was determined; however, there have been no studies examining the reduction rate of stoma closure wounds with PSS, and because we were unable to determine the number of participants, we were forced to conduct an exploratory study. Therefore, there is no clear basis for setting the number of registered cases. Given that our study was designed to investigate the efficacy and safety of NPWT in an exploratory manner, the number of 


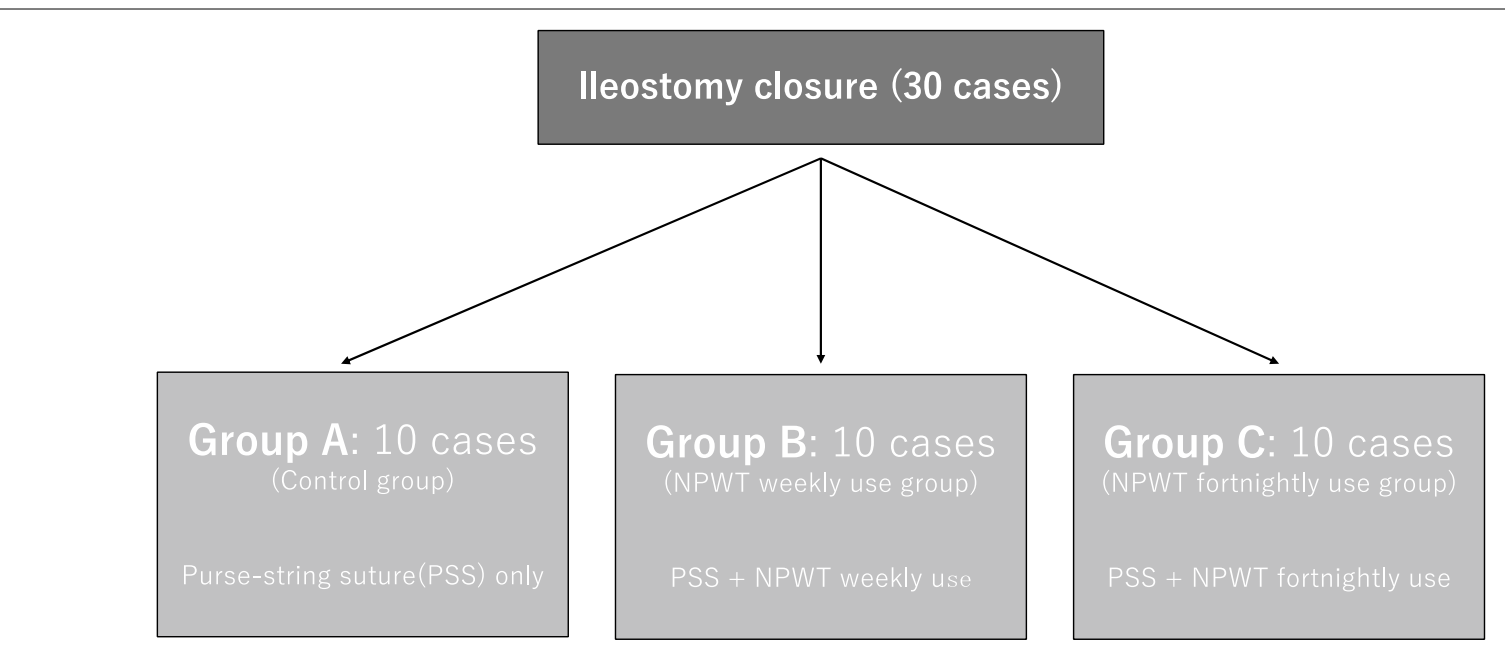

Fig. 4 Study flowchart. The 30 patients enrolled were divided into the groups

\begin{tabular}{|c|c|c|c|}
\hline & Group A & Group B & Group C \\
\hline POD 1 & Start gauze dressing & $\begin{array}{l}\text { Start NPWT } \\
\text { (insert the form) }\end{array}$ & $\begin{array}{l}\text { Start NPWT } \\
\text { (insert the form) }\end{array}$ \\
\hline \multicolumn{4}{|l|}{ POD 2} \\
\hline POD 3 & & $\begin{array}{l}\text { Change NPWT dressing } \\
\text { (remove the form) }\end{array}$ & $\begin{array}{c}\text { Change NPWT dressing } \\
\text { (remove the form) }\end{array}$ \\
\hline \multicolumn{4}{|l|}{ POD 4} \\
\hline \multicolumn{4}{|l|}{ POD 5} \\
\hline \multicolumn{4}{|l|}{ POD 6} \\
\hline POD 7 & & $\begin{array}{l}\text { Remove NPWT dressing } \\
\text { Start gauze dressing }\end{array}$ & Change NPWT dressing \\
\hline \multicolumn{4}{|l|}{ POD 8} \\
\hline \multicolumn{4}{|l|}{ POD 9} \\
\hline POD 10 & & & Change NPWT dressing \\
\hline \multicolumn{4}{|l|}{ POD 11} \\
\hline \multicolumn{4}{|l|}{ POD 12} \\
\hline \multicolumn{4}{|l|}{ POD 13} \\
\hline POD 14 & & & $\begin{array}{l}\text { Remove NPWT dressing } \\
\text { Start gauze dressing }\end{array}$ \\
\hline \multicolumn{4}{|l|}{ POD 15} \\
\hline POD 16 & & & \\
\hline
\end{tabular}

Fig. 5 Study protocol. The treatment schedule for each of the three groups 
enrolled cases was set at 30 (10 cases for each intervention group; Figs. 4 and 5).

\section{Results}

Five university hospitals agreed to participate in this study. However, the approval of the ethics committee of each institution was not obtained until study initiation; therefore, the study was conducted only at three institutions. Thirty patients from three university hospitals were enrolled in the study; there were no statistical differences in demographic and clinical characteristics between the groups (Table 1). All the patients enrolled in this study had an American Society of Anesthesiologists class I or II classification; none of them had undergone preoperative chemotherapy or radiotherapy, and none was diagnosed with renal failure. Preoperative diseases that may have affected the outcome were hypertension in eight cases (Group A: three, Group B: three, Group C: two), hypercholesterolemia in three (Group A: one, Group B: zero, Group C: two), ischemic heart disease in one (Group A: zero, Group B: one, Group C: zero), and cerebral infarction in one (Group A: zero, Group B: zero,

Table 1 Patients' demographic and clinical characteristics

\begin{tabular}{|c|c|c|c|c|}
\hline Median [range] & Group A & Group B & Group C & p-value \\
\hline $\begin{array}{l}\text { Institutions, I:II:III } \\
\text { Sex, M:F }\end{array}$ & $\begin{array}{l}6: 4: 0 \\
6: 4\end{array}$ & $\begin{array}{l}6: 1: 3 \\
8: 2\end{array}$ & $\begin{array}{l}6: 3: 1 \\
4: 6\end{array}$ & 0.248 \\
\hline Age & $64[48-82]$ & $69[48-75]$ & $70[43-84]$ & 0.846 \\
\hline Height (cm) & $162.5[148.0-181.0]$ & $165.0[153.0-176.1]$ & $157.7[138.0-175.0]$ & 0.325 \\
\hline Weight (kg) & $59.2[36.0-84.6]$ & $59.6[46.4-69.8]$ & $48.8[39.8-73.9]$ & 0.161 \\
\hline BMI $\left(\mathrm{kg} / \mathrm{m}^{2}\right)$ & $22.2[16.4-30.1]$ & $21.8[16.5-23.8]$ & $20.3[17.0-26.2]$ & 0.394 \\
\hline Thickness of subcutaneous fat (cm) & $1.7[0.3-2.8]$ & $2.1[0.9-3.2]$ & $2.0[0.8-3.8]$ & 0.606 \\
\hline Area of the ileostomy opening $\left(\mathrm{cm}^{2}\right)$ & $9.8[6.3-13.5]$ & $11.7[7.3-25.0]$ & $7.5[3.4-31.5]$ & 0.224 \\
\hline Height of the ileostomy stoma (cm) & $2.0[1.0-3.0]$ & $1.5[1.0-3.5]$ & $2.0[1.3-2.2]$ & 0.284 \\
\hline
\end{tabular}

Institution I: Toho University Omori Medical Center, II: Juntendo University Hospital, III: Kyorin University Hospital. POD postoperative day

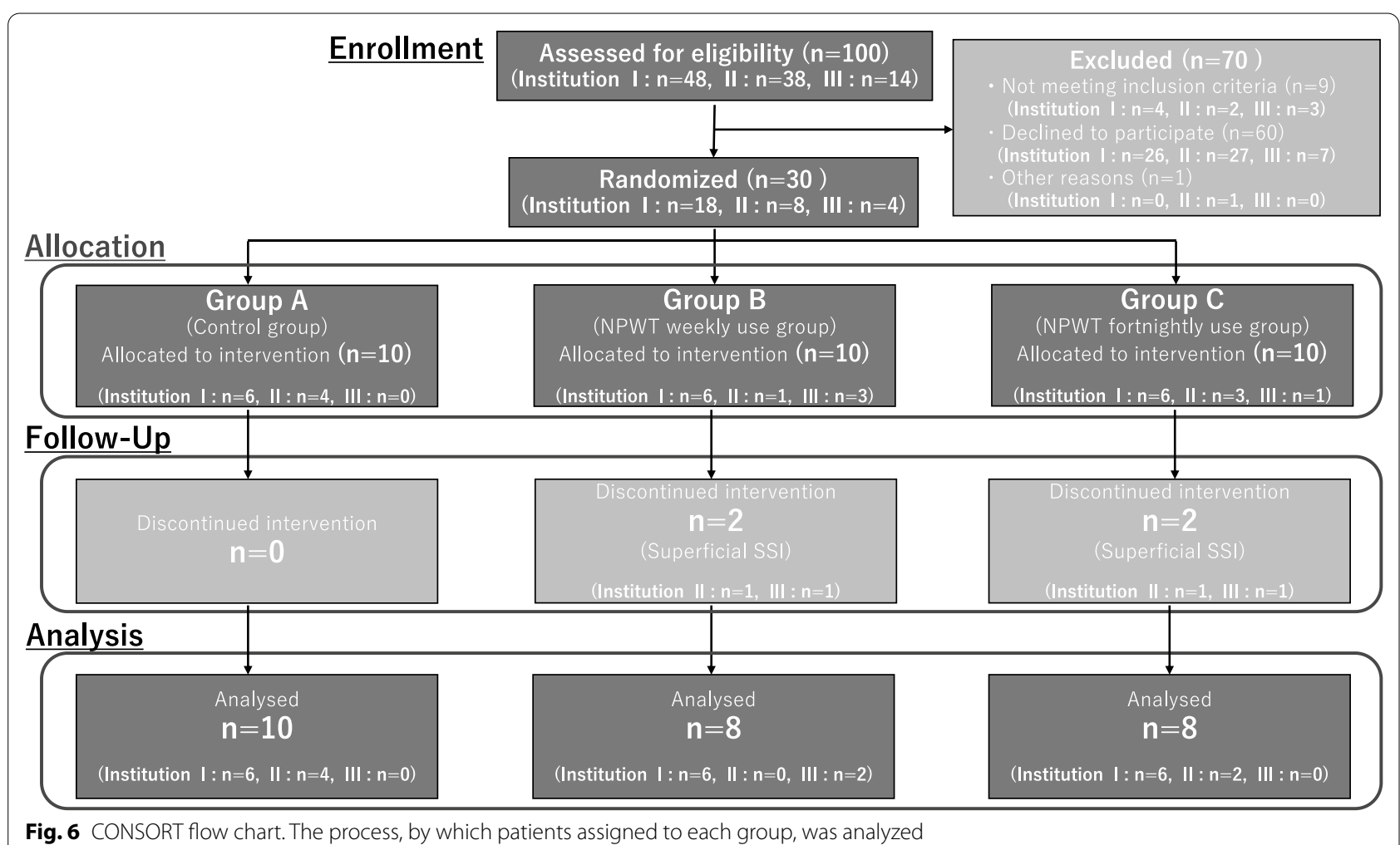

Fig. 6 CONSORT flow chart. The process, by which patients assigned to each group, was analyzed 
Table 2 Postoperative complications

\begin{tabular}{lllll}
\hline & Group A & Group B & Group C & p-value \\
\hline Superficial incisional SSI, $n(\%)$ & $0(0)$ & $2(20)$ & $2(20)$ & 0.507
\end{tabular}

SSI surgical site infection

Group C: one). No significant difference was observed in the baseline characteristics among Groups A, B, and C. The CONSORT flow chart is presented in Fig. 6 , and the postoperative complications are shown in Table 2 . The overall incidence rate of wound infection in the cohort was $13.3 \%$ ( $4 / 30$ cases: 0,2 , and 2 cases in Groups A, B, and $C$, respectively). All SSIs were superficial SSIs.

Given that the patients with SSIs $(n=4)$ were not suited for comparison with those without SSIs, they were excluded from the other study endpoints. On POD 3, the average wound reduction rate was $32.3 \%$ (29.4\%, 40.5\%, and 40.7\% in Groups A, B, and C, respectively), and no significant difference was found between Group A (control) and Groups B and C (Group A vs B: $p=0.278$, Group A vs $C: p=0.284$ ). On POD 7 , the average wound reduction rate was $31.4 \%$ (Group A: $48.4 \%$, Group B: 66.1\%, Group C: $65.9 \%)$. Compared with Group A, Group B showed a significant increase in the wound reduction rate $(\mathrm{p}=0.049)$. There was no significant difference in the wound reduction rate between Groups A and $\mathrm{C}$. Although Group $\mathrm{C}$ demonstrated an increase in the wound reduction rate, the difference was not significant $(\mathrm{p}=0.075)$. On POD 10 , the average wound reduction rate was $67.2 \%$ (58.2\%, 78.6\%, and 69.2\% in Groups $\mathrm{A}, \mathrm{B}$, and $\mathrm{C}$, respectively), and compared with Group A, Group B showed a significant increase in the wound reduction rate $(p=0.011)$; while there was no significant difference between Groups A and C $(\mathrm{p}=0.173)$. On POD 14 , the average wound reduction rate was $72.5 \%(71.0 \%$, 84.4\%, and 76.9\% in Group A, B, and C, respectively), and

Table 3 Average wound reduction rate for each institution

\begin{tabular}{lcccc}
\hline (\%) & POD 3 & POD 7 & POD 10 & POD 14 \\
\hline A: Institution I & 36.1 & 56.1 & 64.0 & 77.9 \\
II & 19.2 & 36.8 & 46.7 & 60.7 \\
III & - & - & - & - \\
p-value & 0.318 & 0.182 & 0.194 & 0.206 \\
B: Institution I & 44.4 & 71.2 & 77.9 & 87.5 \\
II & - & - & - & - \\
III & 28.9 & 50.9 & 80.6 & 74.9 \\
p-value & 0.105 & 0.030 & 0.359 & 0.295 \\
C: Institution I & 32.9 & 58.6 & 69.0 & 77.6 \\
II & 74.0 & 87.8 & 69.8 & 53.8 \\
III & - & - & - & - \\
p-value & 0.039 & 0.010 & 0.490 & 0.157 \\
\hline
\end{tabular}

Institution I: Toho University Omori Medical Center, II: Juntendo University Hospital, III: Kyorin University Hospital. POD postoperative day

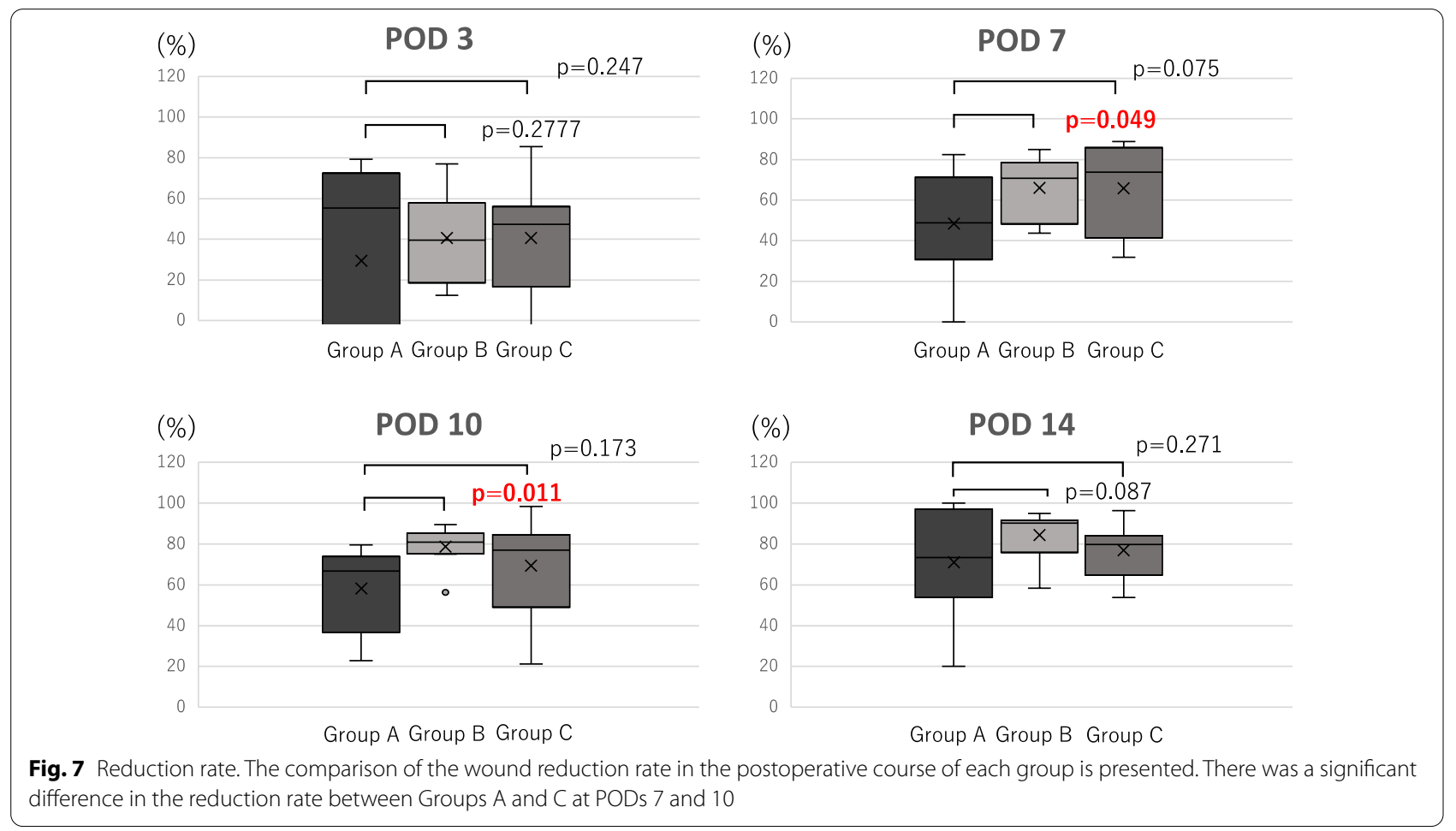


no significant difference was found between the groups (Group A vs. B, p=0.087; Group A vs. C, p=0.271) (Fig. 7). In addition, there were no significant differences in the wound reduction rates between institutions in most situations (Table 3).

The average wound healing duration was 26.7 days (Group A: 26.4 days, Group B: 23.1 days, Group C: 30.6 days), and no significant difference was found among the three groups (Group A vs. B, p $=0.2448$; Group A vs. $C, p=0.2946$ ) (Fig. 8). In addition, common complications in gastrointestinal surgery such as postoperative bleeding and adhesive bowel obstruction, were evaluated; interestingly, no adverse events other than SSIs occurred in patients undergoing NPWT (Groups B and C). The average length of postoperative hospital stay was 16.0 days (Group A: 15.6 days, Group B: 16.6 days, Group C: 15.5 days). No significant difference was observed among the Groups A, B, and C (Group A vs. $B, p=0.3968$; Group A vs. C, $p=0.6888$; Group B vs. $\mathrm{C}, \mathrm{p}=0.3774)$. NPWT was completed during hospitalization in all patients, and no complications occurred in the outpatient setting.

\section{Discussion}

In this study, the wound reduction rate did not differ between the groups on POD 3, whereas it was superior in Group B compared with that in Group A on PODs 7 and 10; no difference was detected on POD 3 possibly because of NPWT use during the inflammatory phase of the wound healing process, which could not provide the expected effects because of the presence of inflammation. In contrast, NPWT use after the end of the inflammatory phase provided notable benefits, including rapid drainage of exudates, promotion of angiogenesis, proliferation of

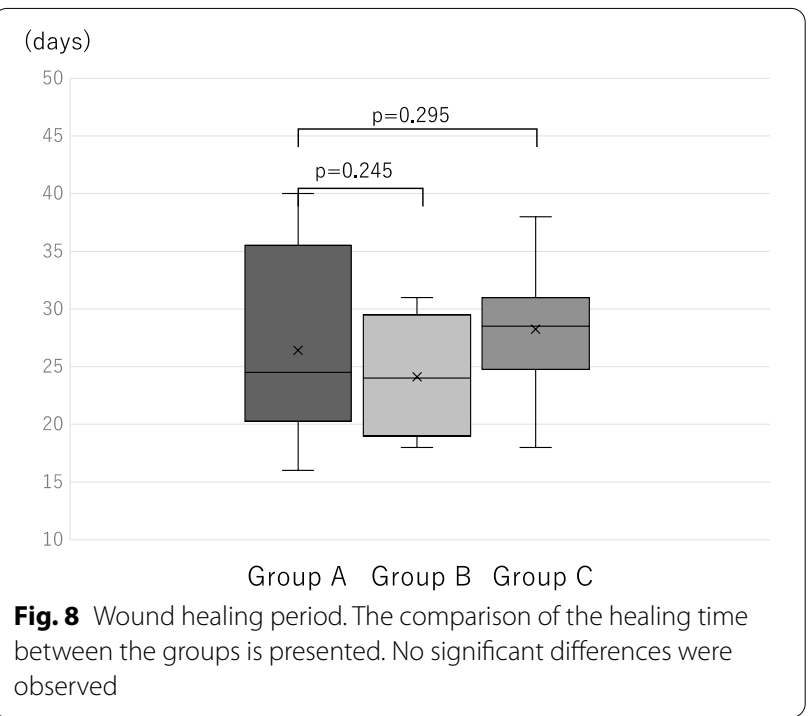

tissue, and reduction of physical wound volume by negative pressure, resulting in effective wound reduction on PODs 7 and $10[18,19]$. Although no significant difference was observed between Groups A and C, the wound reduction rate of Group $C$ was similar to that of Group B. Given that Groups B and C were managed in the same manner until POD 7, the absence of a significant difference between the two groups might be attributed to the small number of cases. Although there was an increase in the wound reduction rate on PODs 7 and 10, the wound healing duration did not differ between the groups, suggesting that NPWT is most effective during the proliferative phase of the wound healing process. Furthermore, early recovery from the inflammatory phase and rapid transition to the proliferative and remodeling phases may have a positive impact on the integrity of the wound during healing.

Although the incidence of SSIs following stoma closure is high $(2-41 \%)[1,20]$, PSS, the wound closure method employed to prevent infection, has been reported to greatly reduce this incidence to $0-5 \%$. Therefore, the incidence of SSIs is not a suitable primary endpoint for phase II studies. Several retrospective reports have suggested that the combination of PSS and NPWT may lead to a shorter wound healing time [16]; however, no difference was found in a previous randomized controlled trial [17]. It is difficult to prove the benefit of NPWT in terms of wound healing time; therefore, the primary endpoint in this study was the wound reduction rate. Bleeding from the wound may cause obstruction of the NPWT dressings and worsen the drainage of exudate; therefore, the dressing was applied the day after surgery, after confirming the absence of bleeding. Although the dressings for the NPWT used in this study were designed to be used for 7 days, they were changed in a shorter period of time as it was necessary to monitor for complications such as bleeding and infection.

In this study, the incidence rate was as high as $13.3 \%$, despite the exclusion of high-risk cases. PSS reduces the rate of infection by opening and draining the wound. Given that an open wound takes time to heal, NPWT is used to promote granulation and to shorten the wound healing duration [10]. Conversely, there has been a longstanding concern that closing the wound might lead to the development of infections. In this study, SSIs only occurred in Groups B and C that underwent NPWT, suggesting that SSIs are associated with closed dressings. We used the PICO Single Use Negative Pressure Wound Therapy System because the procedure is relatively simple and can be used in an outpatient setting; however, infections occurred only in patients who underwent NPWT, which is plausibly explained by the inability of the multi-layered dressing to absorb viscous exudates. If 
NPWT had been performed with a higher pressure and direct foam aspiration, such as with the RENASYS System (Smith \& Nephew Co. Ltd.) or the ActiV.A.C. Therapy System (3 M, Saint Paul, MN, USA) the patient may have been successfully treated without infection. These NPWT types are common for open wounds; they are different from the PICO system used in this study. This potential difference needs to be investigated in future studies.

We found that the wound infections occurred only in certain institutions, indicating that the cause of SSIs in the NPWT group may have been attributed to unfamiliarity of the staff at some institutions, and that the assessment of wound infections may not have been standardized in each institution. Moreover, there may have been a risk of detection bias because the assessment of wounds was not structured, and the treatment received was not blinded. The importance of cautiously using NPWT for wound infections was reconfirmed, and further validation is required after the standardization of wound dressing procedures and wound assessment.

Postoperative complications, all of which were superficial SSIs, were observed in four of the 20 patients who underwent NPWT. Although delayed wound healing was observed, wound closure was observed in all patients without prolongation of hospital stay; thus, the safety of the treatment was proven in this study. We observed a significant difference in the wound reduction rates on PODs 7 and 10. The PICO dressings require replacement every 7 days, suggesting that the optimal duration of NPWT use is from PODs 3 to 10; thus, these days may thus represent the most effective duration of NPWT use for wound reduction.

This study had some limitations, which include its small sample size (30 cases); absence of certain preoperative and perioperative characteristics, such as smoking habits; use of only one type of NPWT system; and the fact that wound assessment was subjective and not blinded. This study reported a significant difference in the wound reduction rate between Groups A and B on POD 7; therefore, the mean difference and standard deviation between the two groups were calculated from these data (mean difference between the two groups: 15.6, standard deviation: 23.6). The sample size, which was calculated using a one-tailed test with a significance level of 0.05 and power of 0.8 , was 29 in each group. The power, which was calculated using a one-tailed test with a sample size of 10 cases in each group according to the mean difference and standard deviation between the two groups, was 0.43 . Although the sample size in this study was not sufficient, it was considered to be within the acceptable range.

The results of this study were uncertain due to the aforementioned limitations; however, there have been no similar multicenter, prospective studies, conducted to date. Therefore, this study may serve as a stepping stone toward evaluating the role of NPWT for the wound of ileostomy closure.

\section{Conclusion}

The most effective duration of NPWT use for ileostomy closure with PSS in terms of the maximum wound reduction rate was from POD 3 to 10. Although NPWT reduced the wound size, it did not shorten the wound healing duration. Additionally, the procedure should be performed with appropriate infection control measures, such as occasional removal of the dressings to monitor the wound. Further studies are needed to confirm our findings.

\section{Abbreviations}

NPWT: Negative pressure wound therapy; POD: Postoperative day; PSS: Pursestring suture; SSI: Surgical site infections.

\section{Acknowledgements}

None.

\section{Authors' contributions}

$\mathrm{KF}$ is the principal investigator of this study. KS organized and supervised this study. KE was involved in devising the research methodology and design. MG, YN, and MK was in charge of wound care. YS, ST, AE, MT, and KI performed wound management as WOCN. HM, TM, and ES assisted in writing this paper. KN was responsible for the safety management of this study. NO was responsible for the monitoring and auditing of this study. KT was responsible for the statistical analysis of this study. All authors read and approved the final manuscript.

\section{Funding}

Not applicable.

\section{Availability of data and materials}

The datasets used and/or analyzed during the current study are available from the corresponding author on reasonable request.

\section{Declarations}

Ethics approval and consent to participate

All study protocols were approved by the institutional review board of Kyorin University, School of Medicine (Approval number: 724) and were performed in accordance with the relevant guidelines. Written informed consent and approval for the use of patient data were obtained before surgery. The study protocol was registered prospectively in the University Hospital Medicine Information Network Clinical Trials Registry (trial registration number: UMIN000032174) on 10/04/2018

\section{Consent for publication}

Not applicable.

\section{Competing interests}

The authors declare that they have no competing interests.

\section{Author details}

${ }^{1}$ Department of Surgery, Kyorin University School of Medicine, 6-20-2, Shinkawa, Mitaka city, Tokyo 181-8611, Japan. ${ }^{2}$ Department of General and Gastroenterological Surgery, Toho University Omori Medical Center, Tokyo, Japan. ${ }^{3}$ WOCN, Department of Nursing, Toho University Omori Medical Center, Tokyo, Japan. ${ }^{4}$ Department of Coloproctological Surgery, Juntendo University School of Medicine, Tokyo, Japan. ${ }^{5}$ WOCN, Department of Nursing, Juntendo 
University School of Medicine, Tokyo, Japan. ${ }^{6}$ WOCN, Department of Nursing, The Jikei University School of Medicine, Tokyo, Japan. ${ }^{7}$ WOCN, Department of Nursing, Kyorin University School of Medicine, Tokyo, Japan. ${ }^{8}$ WOCN, Department of Nursing, Teikyo University School of Medicine, Tokyo, Japan. ${ }^{9}$ Department of Paramedics, Kyorin University Faculty of Health Sciences, Tokyo, Japan. ${ }^{10}$ Department of Plastic, Reconstructive and Aesthetic Surgery, Kyorin University School of Medicine, Tokyo, Japan. ${ }^{11}$ Department of Health \& Welfare, Kyorin University Faculty of Health Sciences, Tokyo, Japan. ${ }^{12}$ Department of Surgery, The Jikei University School of Medicine, Tokyo, Japan.

${ }^{13}$ Department of Surgery, Teikyo University School of Medicine, Tokyo, Japan.

Received: 20 January 2021 Accepted: 20 December 2021

Published online: 28 December 2021

\section{References}

1. Hackam DJ, Rotstein OD. Stoma closure and wound infection: an evaluation of risk factors. Can J Surg. 1995;38:144-8.

2. Milanchi S, Nasseri Y, Kidner T, Fleshner P. Wound infection after ileostomy closure can be eliminated by circumferential subcuticular wound approximation. Dis Colon Rectum. 2009;52:469-74.

3. Klink CD, Wunschmann M, Binnebosel M, Alizai HP, Lambertz A, Boehm $G$, et al. Influence of skin closure technique on surgical site infection after loop ileostomy reversal; retrospective cohort study. Int J Surg. 2013;11:1123-5.

4. Banerjee A. Pursestring skin closure after stoma reversal. Dis Colon Rectum. 1997:40:993-4

5. Sutton CD, Williams N, Marshall LJ, Lloyd G, Thomas WM. A technique for wound closure that minimizes sepsis after stoma closure. ANZ J Surg. 2002;72:766-7.

6. Amamo K, Ishida H, Kumamoto K, Okada N, Hatano S, Chika N, et al. Purse-string approximation vs. primary closure with a drain for stoma reversal surgery: results of a randomized clinical trial. Surg Today. 2019:49:231-7.

7. Ban KA, Minei JP, Laronga C, Harbrecht BG, Jensen EH, Fry DE, et al. American College of Surgeons and Surgical Infection Society: surgical site infection guidelines, 2016 update. J Am Coll Surg. 2017;224:59-74.

8. Armstrong DG, Lavery LA, Diabetic Foot Study Consortium. Negative pressure wound therapy after partial diabetic foot amputation: a multicentre, randomized controlled trial. Lancet. 2005;366:1704-10.

9. Raja SG, Berg GA. Should vacuum-assisted closure therapy be routinely used for management of deep sternal wound infection after cardiac surgery? Interact Cardiovasc Thorac Surg. 2007;6:523-7.

10. Morykwas MJ, Argenta LC, Shelton-Brown El, McGuirt W. Vacuum-assisted closure: a new method for wound control and treatment: animal studies and basic foundation. Ann Plast Surg. 1997;38:553-62.

11. Sinha K, Chauhan VD, Maheshwari R, Chauhan N, Rajan M, Agrawal A. Vacuum assisted closure therapy versus standard wound therapy for open musculoskeletal injuries. Adv Orthop. 2013. https://doi.org/10.1155/ 2013/245940.

12. Hyldig N, Birke-Sorensen H, Kruse M, Vinter C, Joergensen JS, Sorensen JA, et al. Meta-analysis of negative-pressure wound therapy for closed surgical incisions. Br J Surg. 2016;103:477-86.

13. Azuma Y, Watanabe K, Kitamura Y, Maki H, Satou S, Nagao A, et al. Effect of pressure wound therapy for stoma closure. J Jpn Soc Surg Infect. 2017;14:249-51 (in Japanese).

14. Funahashi K, Nagashima Y, Goto M, Kaneko T, Sakai T, Kagami T, et al. Techniques for wound closure and wound management to prevent surgical site infection following stoma reversal. J Jpn Surg Soc. 2019;120:51-4 ([in Japanese).

15. Obeid N, Sharma E, Dunstan M, Nisar P, Trivedi P, Madani R, et al. Negative pressure therapy for stoma closure sites - a nonrandomized case control study. Int J Colorectal Dis. 2021;36:161-7.

16. Naito A, Murata K, Kagawa Y, Kawai K, Kuwahara R, Masuzawa T, et al. Wound management in stoma closure using negative pressure wound therapy. Nippon Daicho Komonbyo Gakkai Zasshi (J Jpn Soc Coloproctol). 2018;71:173-6 (in Japanese).

17. Uchino M, Hirose K, Bando T, Chohno T, Takesue Y, Ikeuchi H. Randomized controlled trial of prophylactic negative-pressure wound therapy at ostomy closure for prevention of delayed wound healing and surgical site infection in patients with ulcerative colitis. Dig Surg. 2016:33:449-54.

18. López N, Cervero S, Jiménez MJ, Sánchez JF. Cellular characterization of wound exudate as a predictor of wound healing phases. Wounds. 2014;26:101-7.

19. Hourigan LA, Hourigan L, Linfoot JA, Linfoot J, Chung KK, Chung K, et al. Loss of protein, immunoglobulins, and electrolytes in exudates from negative pressure wound therapy. Nutr Clin Pract. 2010;25:510-6.

20. Wong KS, Remizi FH, Gorgun E, Arrigain S, Church JM, Preen M, et al. Loop ileostomy closure after restorative proctocolectomy: outcome on 1,504 patients. Dis Colon Rectum. 2005;48:243-50.

\section{Publisher's Note}

Springer Nature remains neutral with regard to jurisdictional claims in published maps and institutional affiliations.

Ready to submit your research? Choose BMC and benefit from:

- fast, convenient online submission

- thorough peer review by experienced researchers in your field

- rapid publication on acceptance

- support for research data, including large and complex data types

- gold Open Access which fosters wider collaboration and increased citations

- maximum visibility for your research: over 100M website views per year

At BMC, research is always in progress.

Learn more biomedcentral.com/submissions 